\title{
Reservatórios animais da leptospirose: Uma revisão bibliográfica
}

\author{
Stefan Vilges de Oliveira*, Maria de Lourdes Nobre Simões Arsky*, \\ Eduardo Pacheco de Caldas**
}

Resumo: Através de uma revisão bibliográfica, apresentamos algumas das principais espécies animais envolvidas no ciclo de transmissão da leptospirose. Foram lidos 80 trabalhos publicados, a busca se deu através de portais indexadores, bibliotecas, livros e informes técnicos que citam como descritores as palavras lepstospiras em animais; sorovares de leptospiras e leptospirose em animais. Assim, relatamos os achados de leptospira em espécies domésticas, de criação, sinantrópicas e silvestres. 0 conhecimento dos sorovares prevalentes, bem como dos hospedeiros que participam do ciclo da leptospirose é uma estratégia de grande importância que deve ser adotada para o entendimento epidemiológico da doença.

Descritores: Sorovares de Leptospiras, Reservatórios Animais, Leptospirose Animal.

Animal reservoirs of leptospirosis: a literature review

Abstract: Through a literature review, we present some of the main species involved in the transmission cycle of leptospirosis. Were read 80 articles published, the research was done through portals of Indexers, libraries, books and technical reports mentioning the words as descriptors lepstospiras in animals; serovars of leptospires and leptospirosis in animals. Thus, we report the results of leptospira in domestic species of creation, synanthropic and wild. The knowledge of prevalent serovars and reservoirs that participate in the cycle of leptospirosis is an important strategy to be adopted for understanding the epidemiology of the disease.

Descriptors: Serovars of Leptospires, Animal Reservoirs, Animal Leptospirosis.

*Biólogo pela Universidade da Região da Campanha (URCAMP), Caçapava do Sul, RS, Brasil. Mestrando em Medicina Tropical na Universidade de Brasília (UnB), Brasília, DF, Brasil.

**Funcionário na Unidade Técnica de Vigilância de Zoonoses da Coordenação Geral de Doenças Transmissíveis, Departamento de Vigilância das Doenças Transmissíveis, Secretaria de Vigilância em Saúde, Ministério da Saúde, Brasilia, DF, Brasil. 


\section{Introdução}

A leptospirose é uma zoonose de ampla distribuição, com significativo impacto social, econômico e sanitário. Esta enfermidade acomete o ser humano e praticamente todos os animais domésticos e selvagens, entre os quais se destacam os carnívoros, roedores, primatas e marsupiais, que podem se tornar portadores e contribuírem para a disseminação do microrganismo na natureza ${ }^{1}$.

As leptospiras foram detectadas em praticamente todos os países que realizaram investigações epidemiológicas e está mundialmente distribuída ${ }^{2}$. Sua ocorrência tem forte associação com períodos de elevados índices pluviométricos ${ }^{1,3}$.

Em condições favoráveis e na presença dos hospedeiros adequados, as leptospiras podem persistir por semanas ou meses no ambiente, principalmente em regiões tropicais e subtropicais ${ }^{3}$.

Em regiões de clima seco, infecções acidentais ocorrem próximas a águas represadas com alta concentração de animais. Em regiões temperadas, às infecções são sazonais e ocorrem com maior frequência nos meses chuvosos ${ }^{4}$.

Na América Latina, na África e na Ásia, os casos de leptospirose humana atingem principalmente a população de baixo poder aquisitivo, e seu controle apóia-se em melhoria de saneamento básico, água, esgoto, colheita e disposição de lixo e melhores condições de moradia 5,6

0 agente etiológico da leptospirose pertence à ordem Spirochaetales, família Leptospiraceae, gênero Leptospira. Segundo a classificação taxonômica clássica, com base em sorogrupos e sorovares e na patogenicidade, as leptospiras podem ser divididas em dois grandes grupos: patogênicas e saprófitas. As patogênicas, que podem infectar o homem e os animais são: Leptospira interrogans, L. borgpetersenii, L. inadai, L. kirschneri, L. noguchii, L. weillii e L. santarosai; possuem mais de 200 sorovares agrupados em 23 sorogrupos. As espécies saprófitas de vida livre são: L. biflexa, L. wolbachii e L. hollandia; possuem 38 sorovares agrupados em seis sorogrupos, estas são encontradas principalmente em água doce, e os registros de infecção ao homem e animais são raros 5,1,7. A variante sorológica ou sorovar é a unidade taxonômica do gênero.

O diagnóstico laboratorial da leptospirose é feito a partir de provas bacteriológicas e sorológicas específicas, e de provas inespecíficas, principalmente de natureza bioquímica ${ }^{6}$. Entre as provas específicas mais utilizadas temos a pesquisa direta de anticorpos, que podem ser detectados no sangue aproximadamente de 5 a 7 dias após 0 início dos sintomas 0 cultivo do agente etiológico e 0 isolamento ${ }^{8}$.

Os animais acometidos pela infecção da leptospirose podem ser passíveis a duas modalidades: doentes portadores convalescentes e os portadores assintomáticos. A infecção por Leptospira spp ocorre através das mucosas ou de lesões de pele, seguindo-se da sua multiplicação no sangue e praticamente em todos os órgãos e tecidos. Nos animais que conseguem sobreviver à fase aguda da leptospirose, os microrganismos alcançam o sistema renal e passam a ser excretados pela urina por períodos de tempo variados, caracterizandoo como portador convalescente 9 .

Saúde (Santa Maria), v.39, n.1, p. 9-20, 2013. Reservatórios animais da leptospirose: Uma revisão bibliográica

Ressalta-se que aos portadores é atribuída a maior parcela de contribuição pela manutenção dos focos de leptospirose, tendo em vista a longa duração desta condição (meses ou anos) e a ampla facilidade de deslocamento atribuída a estes animais, uma vez que os mesmos podem não apresentar sinais de infecção ${ }^{6}$. 
Desta forma este estudo teve o objetivo de consolidar informações referentes às principais espécies animais envolvidas como reservatórios no ciclo da Leptospirose. E justifica-se pelo fato da literatura científica não dispor, até o momento, de uma revisão completa desta temática.

\section{Materiais e Métodos}

Foi realizada uma revisão na literatura científica para compor o embasamento teórico desta narrativa, utilizaram-se de livros e trabalhos em língua inglesa, espanhola e portuguesa, publicações em periódicos nacionais e internacionais de impacto em saúde pública, alem de dissertações, teses e informes técnicos.

Foram selecionados artigos disponíveis nas seguintes bases de dados: Scientific Electronic Library Online (Scielo), JCM, ACM Journals e Portal Elsevier. Os descritores da pesquisa foram: lepstospiras em animais, sorovares de leptospiras, leptospirose em animais

\section{Resultados e Discussões}

Nesta revisão foram citadas 80 referências, sendo $81 \%$ periódicos, 10,1\% informes técnicos, $7,6 \%$ livros e 1,3\% dissertações e teses.

Em relação aos trabalhos relacionados à leptospirose animal, o maior número de publicações que acessamos foi do ano 2009 (Figura 1).

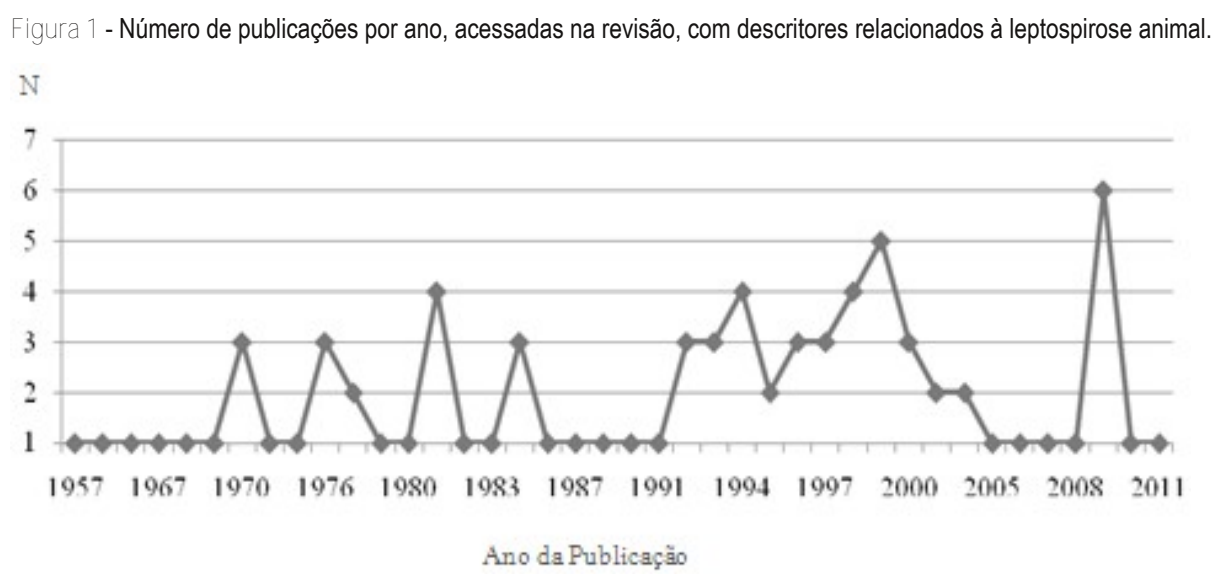

Leptospirose em animais domésticos

A leptospirose canina representa um sério problema sanitário, não só pela gravidade da doença, mas também pelo potencial de contágio ao homem, devido à proximidade estabelecida entre os seres humanos e os cães ${ }^{10}$.

Segundo Alves et al. (2000), os sorovares mais comumente associados à leptospirose canina clássica são o Icterohaemorrahagiae e o Canícola ${ }^{11}$. Alguns destes que têm sido encontrados inclusive no Brasil, infectando cães e causando quadros mórbidos ou infecções benignas são: Pomona, Castellonis, Pyogenes, e Copenhageni 12,13. A prevalência Rev. Saúde (Santa Maria), Santa Maria, v.39, n.1, p. 9-20, Jan./Jul.2013. Oliveira, S. V; ; t al. encontrada em populações caninas brasileiras tem variado entre 10 a $22 \%{ }^{14}$. 
Estudos realizados com felinos domésticos descrevem estes animais como refratários a doença ${ }^{15}$. Entretanto, Langoni et al. (1998) detectou aglutininas anti-Leptospiras em nove amostras de soro de gatos, de várias regiões do país, com títulos de anticorpos, variáveis de 100 a $400 \mathrm{UI}$, consideram-se como reagentes títulos iguais ou superiores a $100 \mathrm{UI} 16,17$. Os sorovares prevalentes foram $4(44,4 \%)$ sorovar Icterohaemorrhagiae, $2(22,2 \%)$ sorovar Patoc, 1 (11,11\%) Canicola, $1(11,11 \%)$ Gryppotyphosa e $1(11,11 \%)$ Andamana.

Leptospirose em animais de criação

Os primeiros estudos sobre a leptospirose bovina no Brasil surgiram no final dos anos 50, a partir dos relatos pioneiros de Freitas et al. (1957) que pela primeira vez identificaram a existência da infecção pelo sorotipo L. pomona em feto abortado em São Paulo ${ }^{18}$.

Nos bovinos, a leptospirose dependendo do sorotipo envolvido e da idade do indivíduo acometido pode provocar abortamentos, infertilidade, anorexia, pirexia, apatia, icterícia, anemia hemolítica, hemoglobinúria, mastite e pode até levar a morte ${ }^{19,20}$.

Com relação a trabalhos realizados no Brasil sobre a ocorrência de leptospirose bovina, Lilenbaum et al. (1996) observou que a partir da década de 70 houve um maior interesse por parte dos pesquisadores ${ }^{21}$. Assim, muitos trabalhos foram publicados em vários Estados e na maior parte havia predominância dos sorotipos Pomona, Wolffi e Sejroe, ressaltando-se que na época não era utilizada na bateria de antígenos o sorotipo Hardjo.

Diversos sorotipos têm sido considerados como causadores de leptospirose em bovinos, predominando os sorotipos Wolffi e Hardjo, muito embora outros sorotipos também tenham ocorrido ${ }^{22,23,24}$.

Ribeiro et al. (1988) analisando infecção por leptospira em bois e cavalos, no Estado de Minas Gerais verificou que os equinos reagiam a mais de um sorotipo, com predominância do sorotipo Canícola (65\%), Castellonis (60\%), Bratislava (55\%) e Pyrogenes (50\%) ${ }^{22}$.

Caldas et al. (1991) pesquisando o comportamento de sorotipos apatogênicos no diagnóstico de triagem da leptospirose em animais constatou os seguintes resultados: bovino (31/500) 62,8\% sorotipos Wolffi, Andamana, Castellonis e Tarassovi; equina (96/200) 45\% Jequitaia, Buenos aires, Castellonis, Autumnalis e Pyrogenes: caprina (102/200/51\%) Castellonis, Autumnalis Buenos aires, e Grippotyphosa; ovina (124/200) 46\% Autumnalis e Buenos aires; canina (123/200) 66,5\% Icterohaemorrhagiae, Autumnalis, Castellonis , Australis ${ }^{25}$.

Em estudos realizados com bubalinos as variantes mais frequentemente encontradas, tem sido, Hardjo, Pomona, Wolffi, 26, 27, 28,29,30.

A leptospirose nos caprinos, nos casos de infecção natural, os sinais mais encontrados são: perda de peso, icterícia, hemoglobinúria, anorexia, letargia e hipertermia, com duração do quadro por 2 a 4 dias; em fêmeas prenhes têm-se observado abortos. Nos casos de doença aguda, pode ocorrer uma alta taxa de mortalidade (superior a 40\%). Nesta espécie, as observações têm-se restringido à frequência de títulos sorológicos em animais aparentemente saudáveis, sendo que os sorovares mais prevalentes são Pomona, Autumnalis, Sejroe, Icterohaemorragiae, Gripotyphosa e Ballum ${ }^{31}$.

Em rebanhos de ovinos estudados no Rio Grande do Sul, a leptospirose apresentou

Saúde (Santa Maria), v.39, n.1, p. 9-20, 2013. Reservatórios animais da leptospirose: Uma revisão

bibliográfica uma prevalência ponderada de 48,7\% (IC 95\%, 35,4-62,0\%) encontrada nas Mesorregiões Sudeste e Sudoeste Rio-Grandense, e em 17 dos 18 municípios sorteados 
probabilisticamente, evidenciou-se uma ampla dispersão das Leptospiras spp na região, corroborando com o trabalho de Santa Rosa e Pestana de Castro (1963) 32. Muito embora exposto a várias sorovariedades de Leptospira spp, a mais prevalente em ovinos é a sorovariedade Hardjo.

Em equinos de diversos países, a sorologia para leptospirose tem se mostrado bastante variável, não demonstrando a ocorrência de um sorovar preferencial. Consequentemente, a incidência de cada sorovar de leptospira varia conforme as características regionais 33 .

Nos suínos os sorovares de leptospiras mais comumente encontrados, infectantes e causadores de doença são: Pomona, Icterohaemorrhagiae, Tarassovi, Canicola, Gryppotyphosa, Bratislava e Muenchen. Dessas, os quatro primeiros já foram isolados de suínos no Brasil ${ }^{34}$.

A leptospirose suína está classificada como uma doença da lista B, no Office International dês Épizooties, grupo ao qual pertencem as doenças transmissíveis de grande importância do ponto de vista sócio-econômico e/ou sanitário, com considerável repercussão no comércio internacional de animais e produtos de origem animal 35,36 .

No Brasil, a leptospirose em suínos tem sido uma das principais causas de falhas reprodutivas em vários Estados, principalmente nas regiões Sul e Sudeste do País ${ }^{37}$.

Leptospirose em animais sinantrópicos e silvestres

Estudos sobre a presença de leptospiras em animais silvestres e sinantrópicos foram realizados em roedores, edentatas, carnívoros, artiodáctilos e primatas, os quais podem atuar como fonte de infecção e potenciais disseminadores dos diferentes sorovares de Leptospira spp $38,39,40,41,42,43,44,45,46,47,48,49,50$

$\mathrm{Na}$ fauna selvagem, os sinais observados são semelhantes aos apresentados por animais domésticos, havendo registros de baixo índice de fertilidade, nascimento de crias fracas, abortamentos e transtornos oculares ${ }^{5}$.

$\mathrm{Na}$ ordem Rodentia, o rato de esgoto (Rattus norvegicus) é considerado como o reservatório do sorovar Icterohaemorrhagiae ${ }^{39}$. No rato d'água (Nectomys squamipes) tem sido demonstrado o sorovar Australis ${ }^{42}$ e no preá (Cavia aperea azarae), o sorovar Icterohaemorrhagiae ${ }^{52,53}$.

No Brasil, as capivaras (Hidrochoerus hidrochoeris) também têm sido estudadas como reservatórios de leptospiras e alguns trabalhos foram realizados para a detecção de leptospiras ${ }^{9}$, sorologia ${ }^{54,55} \mathrm{e}$ isolamento do agente ${ }^{56}$. Os sorovares patogênicos Bratislava e Noguchii têm sido os mais prevalentes.

Em trabalho realizado no Sul do país, a Leptospira isolada dos rins de capivaras, demonstrou amplificações que permitiu concluir que 0 isolado é do sorogrupo Icterohaemorrhagiae. Quanto ao sorovar isolado acredita-se pertencer a Icterohaemorrhagiae ou Copenhageni ${ }^{57}$.

Entre os suídeos selvagens, os javalis (Sus scrofa) representam um potencial risco de disseminação de doenças para espécies silvestres, nativas e para o homem ${ }^{58}$. No continente europeu, agentes etiológicos causadores da leptospirose hemorrágica foram identificados em javalis ${ }^{59}$.

Nos marsupiais da ordem didelphimorfia, os gambás (Didelphis marsupialis e Didelphis

Rev. Saúde (Santa Maria), Santa Maria, v $239, n, 1$ p. 9-20, Jan./Jul.2013. Oliveira, S. V.; et al. 
Icterohaemorrhagiae, Szwajizam e Grippotyphosa ${ }^{60,39,61}$ sugerindo seu potencial reservatório para espiroquetas ${ }^{62}$. Títulos para o sorovar Balcanica foram descritos em opossums (Trichosurus vulpecula) na Nova Zelândia 63,64,65.

Recentemente, na região Sul do RS foram capturados 33 gambás-de-orelha-branca (Didelphis albiventris), isolando-se da urina de um destes animais uma cepa de Leptospira borgpetersenii, a qual foi caracterizada pelo sequenciamento do gene rpoB bacteriano. Quando esta cepa foi incluída na técnica MAT (Microscopic agglutination test) foram observados altos títulos de anticorpos aglutinantes de soros caninos, sugerindo uma relação epidemiológica entre os gambás de orelha branca e os cães domiciliados ${ }^{66}$.

Os guaxinins americanos (Procyon lotor), respectivos representantes da família Procyonidae são considerados sinantrópicos na América do Norte e foram descritos como potenciais reservatórios de leptospiras. O sorovar Bratislava foi descrito nesta espécie por Mikaellian (1997) 67 e os sorovares Grippotyphosa, Canicola e Icterohaemorrhagiae descritos por Mitchell (1999) 68 .

Entre os mustelídeos, os furões (Galicts cuja) demonstraram-se suscetíveis à infecção por leptospirose e há registros de casos pelos sorovares Grippotyphosa, Icterohaemorrhagiae, Pomona e Sejroe ${ }^{69}$.

Em um estudo realizado por Baulu (1987) ${ }^{70} \mathrm{em}$ Barbados com macacos verde africano (Cercopithecus aethiops sebaeus) de vida livre foi realizada a Soroaglutinação Microscópica (SAM), e os animais mostraram-se sororeagentes para a Leptospira spp.

No entanto, dos estudos que se têm conhecimento com primatas de vida livre, a baixa incidência da leptospirose na natureza está associada ao comportamento e hábitos arborícolas dos primatas neotropicais, o que reduz a exposição a agentes contaminantes no solo e contato com roedores ${ }^{71}$.

No Pantanal Sul-Mato-Grossense em um estudo realizado com animais silvestres, através de técnicas sorológicas e moleculares revelaram-se sorovares predominantes entre as espécies: Pomona e Butembo para veado campeiro (O. bezoarticus); Hardjobovis para cachorro do mato (C. thous), Icterohaemorrhagiae para jaguatirica (L. pardalis) e T. panchyrus e Pomona para quati (N. nasua). A frequência encontrada por meio da soroaglutinação microscópica (SAM) e do ensaio da Reação da Polimerase em Cadeia (PCR) foram respectivamente $10,2 \%$ e 15,38 para T. pachyrus; $34,21 \%$ e $38,46 \%$ para C. thous; $34,09 \%$ e $28,12 \%$ para $N$. nasua; $5,9 \%$ e 2,4\% para O. bezouarticus; e $14,28 \%$ para L. pardalis, demonstrando que a leptospira circula entre os animais silvestres desta regiãa ${ }^{72}$.

Tatus também foram soroprevalentes para leptospirose e demonstraram-se como risco potencial de transmissão a seres humanos, haja visto que em muitas regiões do país são utilizados como fonte proteica na alimentação humana ${ }^{73}$.

Morcegos (Desmodus roduntus) do estado de São Paulo apresentaram títulos com uma prevalência de 7,8\%, apontando a possivel importância desses animais como reservatórios de leptospiras ${ }^{74}$.

Além disso, um caso de leptospirose humana foi registrado relacionado a um morcego como possivel fonte de infecção em Chicago, EUA ${ }^{75}$.

Em um estudo realizado com 343 morcegos capturados em diferentes áreas de São

Saúde (Santa Maria), v.39, n.1, p. 9-20, 2013. Reservatórios animais da leptospirose: Uma revisão bibliográica ISSN 2236-5834 Paulo, foi confirmada a positividade para leptospiras patogênicas pela PCR, utilizando amostras de tecidos renais, o que sugere que os morcegos podem atuar como reservatórios na cidade de São Paulo ${ }^{76}$. 
Fornazari et al. (2001), observaram pelas técnicas de MAT e PCR, a presença de anticorpos em serpentes peçonhentas e não peçonhentas de vida livre e de cativeiro. Em um total de 147 serpentes analisadas, $52(35,4 \%)$ foram positivas para leptospirose pelo MAT. Destas, a jararaca (Bothrops jararaca) apresentou maior soropositividade $(66,7 \%, n=22 / 33)$. 0 sorovar mais prevalente foi o Hardjo prajtino $(88,5 \%, n=46 / 52)$ e os títulos variaram de 100 a 3200. Leptospira interrogans foi identificada por PCR nos rins e no fígado de caiçaca (Bothrops moojeni) e de jararaca-pintada (Bothrops pauloensis), mostrando 100\% e $93 \%$ de identidade, respectivamente ${ }^{77}$.

Alguns estudos relataram a presença da leptospirose acometendo populações cativas de zoológicos, e descrevem óbitos em primatas ${ }^{46,47}$, guanaco (Esgyrn Guanaco) ${ }^{78}$ e ariranhas (Pteronura brasiliensis $)^{79}$.

No Zoológico Municipal de Uberaba, em uma pesquisa de soroaglutinação microscópica em animais e funcionários, foram colhidas amostras de sangue de 166 animais entre mamíferos, répteis, peixes e aves e dos 36 funcionários.

Dos animais estudados, $17(10,24 \%)$ foram reagentes para anticorpos contra Leptospira spp ${ }^{80}$. Os sorovares mais prevalentes foram Canicola, 8 (47,05\%); Icterohaemorrhagiae, 5 $(29,41 \%)$ e Andamana 2 (11,76\%). Na tabela abaixo (Figura 2) observamos os sorogrupos encontrados nos animais estudados 80 .

Figura 2 - Espécies estudadas em pesquisa de soroaglutinação microscópica para leptospirose no Zoológico Municipal de Uberaba (Esteves, 2005).

\begin{tabular}{cc}
\hline Espécie & Sorovar \\
\hline Cachorro-do-mato (Cerdocyon thous) & Grippotyphosa \\
Lobo-Guará (Chrysocyon brachiurus) & Canicola \\
Jaguatirica (Leopardus pardalis) & Andamana e Icterohaemorrhagiae \\
Suçuarana (Puma concolor) & Canicola e Icterohaemorrhagiae \\
Cateto (Tayassu tajacu) & Icterohaemorrhagiae \\
Jabuti (Geochelone spp.) & Andamana \\
Tigre d'água (Trachemys scripta) & Patoc \\
Tilápia (Oreochromis niloticus) & Canicola \\
Rato de telhado (Rattus rattus) & Icterohaemorrhagiae \\
\hline
\end{tabular}

As aves, os felinos domésticos e os funcionários do zoológico foram negativos para os sorovares estudados. Dentre as espécies estudadas, os mamíferos e os peixes apresentaram maior soroprevalência 80.

\section{Conclusão}

Todos os animais são susceptíveis à doença e podem atuar como fonte de infecção. Rev. Saúde (Santa Maria), Santa Maria, v.39, n.1, p. 9-20, Jan./Jul.2013. Conhecer os sorovares prevalentes em uma população e os hospedeiros que permitem a manutenção do ciclo da doença em cada região são estratégias importantes para 0 ISSN 2236-5843 
entendimento epidemiológico da doença, pois as infecções humanas resultam da exposição à urina dos animais portadores.

A prevalência de diferentes sorovares de leptospira dentro de uma população depende dos reservatórios animais presentes nessa região e dos sorovares que eles albergam.

O controle da leptospirose animal deve partir da integração de medidas preventivas instituídas simultaneamente nos três estágios da cadeia de transmissão: 1) fontes de infecção (vertebrados infectados), 2) vias de transmissão (água, solo e fômites contaminados) e 3) suscetíveis (vertebrados não infectados).

Do exposto conclui-se que a leptospirose animal representa um ponto de preocupação para os profissionais envolvidos com a saúde animal e saúde publica veterinária. Investigações sorológicas e isolamento de Leptospiras em animais domésticos e silvestres são importantes para uma melhor compreensão da epidemiologia da doença.

\section{Referências}

1. Acha, PN, Szyfres B. Zoonoses y enfermedades transmisibles comunes al hombre y a los animales. $2 \mathrm{ed}$. Organización Panamericana de la Salud; 1986. 112 p.

2. Pandey R. Microbiologia veterinária: perspectivas clínicas e moleculares. São Paulo: Roca; 1994. p. 17093.

3. Plank R, Dean D. Overview of the epidemiology, microbiology, and pathogenesis of Leptospira spp. in humans. Microbes and Infection, Paris. 2000;2(1):1265-6.

4. Szyfres B. Leptospirosis an animal and public health problem in Latin America and the Caribbean Area. Panamerican Health Organization Bulletin, Washington. 1976; (10):110-25.

5. Faine S. Guidelines for Control of Leptospirosis. Geneva: W.H.0; 1982. 171p.

6. Brasil. Ministério da Saúde. Fundação Nacional da Saúde. Centro nacional de Epidemiologia. Coordenação de Controle de Zoonoses e Animais. Programa nacional de leptospirose. Manual de leptospirose. Centro Nacional de Epidemiologia. Coordenação de controle de Zoonoses e Animais Peçonhentos. 2. ed. Brasília; 1999.

7. Kmety E, Dikken $\mathrm{H}$. Classification of species of the Leptospira interrogans and history of its serovars. In: International Leptospirosis. Leptospira classification with history of serovars. 1992 [Acesso em 2011 Nov 13]. Disponivel em: www.vet.bg.ac.yu/ils/leptoclassif/serolis.htm

8. Brasil. Ministério da Saúde. Secretaria de Vigilância em Saúde. Departamento de Vigilância Epidemiológica. Guia de Vigilância Epidemiológica. $7^{\mathrm{a}}$ ed. Brasília; 2009. 816 p.

9. Vasconcellos SA. Detection of Leptospira in two free living populations of capybaras (Hydrochaeris hydrochaeris) from São Paulo State, Brazil. International Leptospirosis Society Barbados. 2002, p. 62.

10. Organização Mundial da Saúde. Current problems in leptospirosis research. Report of a who expert group. Techn Rep Serv. 1967;(380),1-32.

11. Scanziani E, Calcaterra S, Tagliabue S. Serologic findings in cases of acute leptospirosis in the dog. J Small An Pract. 1994;(35):257-60.

12. Dickeson D, Love DN. A serological survey of dogs, cats and horses in south-eastern Australia for

Saúde (Santa Maria), v.39, n.1, p. 9-20, 2013 Reservatórios animais da leptospirose: Uma revisão

bibliográfica leptospiral antibodies. Austral Vet J. 1993;70(10):389-90.

13. Brihuega B, Hutter E. Incidência de la leptospirosis em caninos de la ciudad de Buenos Aires. Vet Argentina. 1994;11(102):98-101. 
14. Alves CJ, Andrade JSL, Vasconcellos AS, Morals ZM, Azevedo SS, Santos FA. Avaliação dos níveis de aglutininas anti-leptospira em cães no município de Patos - PB, Brasil. Rev Bras Ciênc Vet. 2000;7(1):17-21.

15. Corrêa WM, Corrêa CNM. Enfermidades infecciosas dos mamíferos domésticos. $2^{\text {a }}$ ed. Rio de Janeiro: Medsi; 1992. p. 219-232.

16. Langoni H, Cabral KG, Kronfly CS. Pesquisa de aglutininas anti-leptospíricas em gatos. Rev Clín Vet. 1998;(17):24-6.

17. Bolin CA. Diagnosis of leptospirosis: a reemerging disease of companion animals. Semin. Vet Med Surg (Small Animal). 1996;11(3):166-71.

18. Freitas DC, Lacerda Júnior PMG, Veiga JS, Lacerda JPG. Identificação da leptospirose bovina no Brasil. Revista da Faculdade de Medicina Veterinária e Zootecnia São Paulo. 1957;6(1):81-3.

19. Milner AR, Wilks C, Calvert K. The prevalence of antibodies to members of Leptospira interrogans in cattle. Australian Veterinarian Journal. 1980;56:327-30.

20. Michna SW, Campbel RSF. Leptospirosis in wild animals. Journal of Comparative Pathology. 1970;(8):101-6.

21. Lilenbaum W. Atualização em leptospiroses bovinas. Rev Bras Med Vet. 1996; 18(1):9-13.

22. Ribeiro SCA, Silva PC, Barbosa FC, Gouveia MAV, Oliveira PR, Mamede DO. Levantamento sorológico em dois surtos de leptospirose bovina, em Uberlândia, Triângulo Mineiro. Arq Bras Med Vet Zootec. 1988;40:415-23.

23. Ribeiro SCA, Silva PC, Barbosa FC, Gouveia MAV, Oliveira PR, Mamede DO. Leptospirose no rebanho bovino da Sub-Região de Nhecolândia, Pantanal Mato- Grossense, Brasil. Veterinária Notícias, Uberlândia. 1999;5(1):51-5.

24. Lilenbaum W. Leptospirose em reprodução animal. II Bovinos do Estado do Rio de Janeiro, Brasil. Rev Bras Ciências Vet. 1995;2(1):1-6.

25. Caldas EM, Viegas EA, Massa LFM, Reis R. Comportamento de estirpes apatogênicas no diagnóstico sorológico de leptospirose em animais. Arq Esc Med Vet, UFBA. 1991;14(1):3-24.

26. Santa Rosa CA, Castro AFP, Silva AS. Nove anos de leptospirose no Instituto Biológico de São Paulo. Rev Inst Adolfo Lutz. 1969/1970;29/30:19-27.

27. Girio RJS. Estudo comparativo de quatro estirpes apatogênicas utilizadas em teste de triagem para 0 diagnóstico sorológico da leptospirose em búfalos (Bubalus bubalis) [dissertação]. São Paulo: USP, Instituto de Ciências Biomédicas; 1984.52 p.

28. Langoni H, Del Fava C, Abral, KG. Da Silva AV, Chagas SAP. Epidemiological survey on anti-leptospire aglutinins in buffaloes from Vale do Ribeira, SP, State (Brazil). $5^{\circ}$ World Buffalo Congress; Caserta, Italy; 1997. p. 622-25.

29. Sandoval LA, Arruda NM, Teruya JM. Giorgi W, Amaral LBS, Mazanti MT. Pesquisas em bubalinos: prevalência da brucelose e leptospirose no Estado de São Paulo, Brasil. Arq Inst Biol. 1979;45(11-12):209-12.

30. Giorgi W. Teruya JM, Silva AS. Leptospirose: Resultados das soroaglutinações realizadas no Instituto Biológico de São Paulo durante os anos de 1974/1980. Biológico. 1981;47(11):299-309.

31. Williams CSF. Diseases. In: Gall C. (ed). Goat production. London : Academic; 1981. p. 433-87.

32. Santa Rosa CA, Castro AFP. Presença de aglutininas antileptospiras em soro de ovinos e caprinos no Estado de São Paulo. Arquivos Instituto de Biologia. 1963;30:93-8.

33. Hunter P, Herr S. Leptospirosis. In: Coetzer JAW, Thomson GR, Tustin RC. Infectious diseases of Rev. Saúde (Santa Maria), Santa Maria, v.39, n.1, p. 9-20, Jan./Jul.2013. Livestock. Oxford: Oxford University; 1994. (2). p. 997-1008.

34. Sobestiansky J, Barcellos D, Mores N, Carvalho LF, Oliveira S. Clínica e patologia suína. $2^{\mathrm{a}}$ ed. Goiânia: ISSN 2236-5843 
Universidade Federal de Goiás; 1999. 464 p.

35. Blaha T. Applied veterinary epidemiology. Amsterdam: Elsevier, 1989. 95-103.

36. Perry G, Heardy R. A Scientific Review of Leptospirosis and implications for quarentene policy. Austrália: Canberra; 2000. $115 \mathrm{p}$.

37. Langoni $\mathrm{H}$, Cabral KG, Silva AV, Baldini S. Inquérito soroepidemiológico para leptospirose suína. $7^{0}$ Congresso Brasileiro de Veterinários Especialistas em suínos, Blumenau, SC; 1995. Resumos. Blumenau; 1995. p.153.

38. Mccaughey WJ, Fairlei JS. Leptospirosis in Irish wildlife. Veterinary Record. 1971;89(16):447.

39. Santa Rosa CA, Sulzer CR, Giorgi W, Silva AS, Yanaguita RM, Lobão AO. Leptospirosis in wildlife in Brazil; isolation of a new serotype in the pyrogenes group. American Journal of Veterinary Research. 1975:36(9):1363-5.

40. Al Saad M, Post G. Rodent leptospirosis in Colorado. Journal of Wildlife Diseases. Kansas. 1976;12:31521.

41. Cirone SM, Riemann HP, Rupaner R, Behimer DE, Franti CE. Evaluation of the hemagglutination test for epidemiologic studies of leptospiral antibodies in wild mammals. Journal of Wildlife Diseases, Kansas. 1978;14(2):193-202.

42. Cordeiro F, Sulzer CR, Ramos AA. Leptospira interrogans in several widlife species in Southeast Brazil. Pesquisa Veterinária Brasileira, Rio de Janeiro. 1981;1(1):19-29.

43. Everard COR, Fraser-Chanpong GM, Bhagwandin LJ, Race MW, James AC. Leptospirosis in wildlife from Trinidad and Grenada Journal of Wildlife Diseases, Kansas. 1983;19(3):192-9.

44. Michna SW. Leptospirosis. The Veterinary Record. 1970;86:484-96.

45. Reilly JR, Ferris DH, Hanson LE. Experimental demonstration of the enteric route of infection with Leptospira grippotyphosa in wild carnivores. American Journal of Veterinary Research. 1968;29(9):1849-54.

46. Sá LRM, Teixeira RHF, Loreto C, Catão-Dias JL. Leptospirose em primatas neotropicais. III Congresso e VIII Encontro da Associação Brasileira de Médicos Veterinários de Animais Selvagens, São Pedro, SP; 1999. p.7.

47. Shive RJ, Green SS, Evans BS, Garner FM. Leptospirosis in barbary apes. Journal of American Veterinary Medicine Association. 1969;155(7):1776-8.

48. Shimizu MM. Environmental and biological determinants for the prevalence of leptospirosis among wild small mammal hosts, Island of Hawaii. International Journal of Zoonosis. 1984;(11):173-88.

49. Rim BM, Rim CW, Chang WH, Kakoma I. Leptospirosis serology in korean wild animals. Journal of Wildlife Diseases, Kansas. 1993;29(4):602-3.

50. Hartskeerl RA, Terpstra WJ. Leptospirosis in wild animals. The Veterinary Quarterly. 1996;8(3):149-50.

51. Alvares CJ, Vasconcellos AS, Camargo CRA, Morais ZA. Influência de fatores ambientais sobre a proporção de caprinos soro-reatores para leptospirose em cinco centros de criação do estado da Paraíba, Brasil. Arquivos do Instituto Biológico. São Paulo. 1996;63(2):11-8.

52. Pestana de Castro AF, Santa Rosa CA, Troise C. Preás (Cavia aperea azarae, Lichi.)-(Rodentia: Cavidae) como reservatório de Leptospira em São Paulo. Isolamento de Leptospira icterohaemorrhagiae. Arquivos do Instituto Biológico, São Paulo. 1961;(28):219-23.

53. Silva I. A new leptospiral serotype isolated in Salvador, Bahia State. Rev Microbiol. São Paulo. 1976;73:537.

Saúde (Santa Maria), v.39, n.1, p. 9-20, 2013.

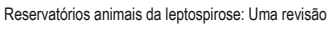

bibliográfica
54. Marvulo MFV, Paula CD, Ferreira PM, Morais ZM, Delbem ACB, Fávero ACM, et al. Detection of Leptospira in two free living populations of capybaras (Hydrochaeris hydrochaeris) from São Paulo state, Brazil. 2002, Proc. 3rd Scient. Meet. Int. Lepospirosis Society, Barbados, s/p. 
55. Ito FH, Vasconcellos AS, Berdnardi F, Nascimento AA, Labruna MB, Arantes IG. Evidencia sorológica de brucelose e leptospirose e parasitismo por ixodídeos em animais silvestres do Pantanal Sul-Matogrossense. Ars Veterinária. 1998;(13):302-10.

56. Paula CD. Isolamento de Leptospira em capivaras (Hydrochaeris hydrochaeris) de vida livre. In: V Congresso Brasileiro da Associação Brasileira de Veterinários de Animais Selvagens. Anais. ABRAVAS, SP; 2001. $25 \mathrm{p}$.

57. Silva EF, Seyffert N, Jouglard SDD, Athanazio DAA, Dellagostin AO, Brod CS. Soroprevalência da infecção leptospiral em capivaras (Hydrochoerus hydrochaeris) abatidas em um frigorífico do Rio Grande do Sul1Pesq. Vet Bras. 2009:29(2):174-6.

58. Gisd. 2007. Global Invasive Species Database. [Acessado em 2011 Out 21].

Disponível em: http://www.issg.org/database/species.

59. Jansen A, Nockler K, Schonberg A, Luge E, Ehlert D, Schneider T.. Wild boars as possible source of hemorrhagic leptospirosis in Berlin, Germany. European Journal of Clinical Microbiology \& Infectious Diseases. 2006;(25):544-6.

60. Hathaway SC, Little TWA, Finch SM, Stevens AE. Leptospiral infection in horses in England: serological study. Vet Rec. 1981:108:396-8.

61. Caldas EM, Fehringer WT, Sampaio MB, Aglutininas anti-leptospiras em Rattus norvegicus e Didelphis marsupialis, em Salvador-Bahia. Arquivos da Escola de Medicina Veterinária, Universidade da Bahia, Salvador. 1992:15(1),43-50.

62. Duhamel GE, Ganley L, Barr BC, Whipple JP, Mathiesen MR, Nordhausen RW, Walker RL, Bargar TW, Van Kruiningen HJ. Intestinal spirochetosis of north american opossums (Didelphis virginianus): a potencial biologic vetor for pathogenic spirochete. Proceedings AAZV and AAWV - Joint Conference. 1998. p. 83-8.

63. Hathaway SC, Marshall RB, Blackmore DK. The serologic and cultural prevalence of Leptospira interrogans serovar balcanica in opossums (Trichosurus vulpecula) in New Zealand. Journal of Wildlife Diseases, Kansas. 1978;14:345-50.

64. Day TD, Wass JR, O'Connor CE, Carey PW Mattheus LR, Pearson AJ. Leptospirosis in bushtail possums: is Leptospira interrogans serovar Balcanica environmentally transmitted? Journal of Wildlife Diseases, Kansas. 1997;33(2):254-60.

65. Day TD, O'Connor CE, Wass JR, Pearson AJ, Mattheus LR. Transmission of Leptospira interrogans serovar Balcanica infection among socially housed bushtail opossums in New Zealand. Journal of Wildlife Diseases, Kansas. 1998;34(3):576-81.

66. Jorge S, Seixas FK, Brihuega B, Dellagostin OA, Samartino L, Fernandes CPH, Brod CS. Role a marsupial (Didelphis albiventris) in maintenance of pathogenic Leptospira: strain isolation, molecular characterization and serological survey. World Association of Veterinary Laboratory Diagnosticians-14th International Symposium, Madrid, Spain; 2009.

67. Mikaelian I, Higgins R, Lequient M, Major M, Lefebure F, Martineau D. Leptospirosis in raccoons in Quebec: 2 case reports and seroprevalence in a recreational area. Canadian Veterinary. 1997;38(7):440-2.

68. Mitchell MA, Hungerford LL, Nixon C, Eske T, Sulliva J, Koerkenmeier R, Dubey JP. Serologic survey for selected infectious disease agents in raccoons from Illinois. Journal of Wildlife Diseases, Kansas. 1999;35(2):347-55.

69. Gobel T. Furões, tratamento das doenças infecciosas. Nosso Clínico - Medicina Veterinária para Animais de Companhia. 2001;4(21):18-24.

70. Baulu J, Everard CO, Everard JD. Leptospires in vervet monkeys (Cercopithecus aethiops Sabaeus) on Barbados. Journal of Wildlife Diseases. 1987;23(1):60-6.

Rev. Saúde (Santa Maria), Santa Maria, v.39, $n$.1 p. 9-20, Jan./Jul.2013. Oliveira, $S . V \cdot$ et a 
71. Reid HA, Herron AJ, Hines ME, Orchard DEA, Altman NH. Leptospirosis in a white-lipped tamarin (Saguinus labiatus). Laboratory of Animal Science. 1993 Jun: 43(3):258-9.

72. Vieira AS. Levantamento de Leptospira spp em animais silvestres do Pantanal Sul-Mato-grossense por meio de técnicas sorologicas e moleculares [dissertação]. UFMS; 2009. 83 p.

73. Da Silva RC, Zetun CB, Bosco Sde M, Bagagli E, Rosa PS, Langoni H. Toxoplasma gondii and Leptospira spp. infection in free-ranging armadillos. Vet Parasitol. 2008;157(3-4):291-3.

74. Zetun CB, Hoffmann JL, Silva RC, Souza LC, Langoni H. Leptospira spp. and Toxoplasma gondii antibodies in vampire bats (Desmodus rotundus) in Botucatu region, SP, Brazil. J Venom Anim Toxins incl Trop Dis. 2009;15(3):546-52.

75. Vashi NA, Reddy P, Wayne DB, Sabin B. Bat-associated leptospirosis. J Gen Intern Med. 2009;25(2):1624.

76. Bessa TAF, Spichler A, Chapola EGB, Husch AC, Almeida MF, Sodré MM, ET AL. The contribution of bats to leptospirosis transmission in São Paulo City, Brazil. Am J Trop Med Hyg. 2010;82(2):315-7.

77. Biscola NP, Fornazari F, Saad E, Richini-Pereira VB, Campagner MV, Langoni H, et al. Serological investigation and PCR in detection of pathogenic leptospires in snakes. Pesq Vet Bras. 2011;3(9).

78. Hodgin C, Schillorn WT, Fayer R, Richter N. Leptospirosis and coccidial infection in a guanaco. Journal of the American Veterinary Medical Association. 1984;185(1): 1442-4.

79. Farias TM, Silva LHR, Pimentel TL. Incidence of leptospirosis in giant otters at the FUNPEB, Brasilia Pole Ecological Foundation. In: Brazil Biennial Conference on the Biology of Marine Mammals. Wailea: The Society of Marine Mammology; 1999. p. 55.

80. Esteves FM, Guerra-Neto G, Girio RJS, Silva-Vergara ML, Carvalho ACFB. Detecção de anticorpos para Leptospira spp. em animais e funcionários do Zoológico Municipal de Uberaba, MG. Arqs Inst. Biológico, São Paulo. 2005;(72):283-8.

\section{Stefan Vilges de Oliveira}

Endereço para correspondência - SCS - Quadra 04, Bloco "A", Edifício Principal, $3^{\circ}$ Andar. Torre B, Brasília, CEP: 70.304-000, DF, Brasil.

E-mail: stefanbio@yahoo.com.br

Currículo Lattes: http://lattes.cnpq.br/8869553181466970

Recebido em 29 de março de 2012.

Aprovado em 14 de janeiro de 2013.

Saúde (Santa Maria), v.39, n.1, p. 9-20, 2013. 